\title{
Does the Presence of Significant Mitral Regurgitation prior to Transcatheter Aortic Valve Implantation for Aortic Stenosis Impact Mortality? - Meta-Analysis and Systematic Review
}

\author{
Ankur Sethi $^{\mathrm{a}}$ Vamsi Kodumuri ${ }^{\mathrm{b}}$ Vinoy Prasad ${ }^{\mathrm{c}}$ Ashok Chaudhary $^{\mathrm{a}}$ \\ James Coromilas ${ }^{\text {a }}$ John Kassotis ${ }^{a}$

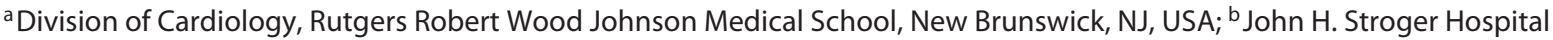 \\ of Cook County, Chicago, IL, USA; ' Division of Cardiology, Loma Linda University, Loma Linda, CA, USA
}

\section{Keywords}

Mitral regurgitation - Aortic stenosis - Transcatheter aortic valve implantation - Transcatheter aortic valve replacement

\begin{abstract}
Background: Mitral regurgitation (MR) is commonly encountered in patients with severe aortic stenosis (AS). However, its independent impact on mortality in patients undergoing transcatheter aortic valve implantation (TAVI) has not been established. Methods: We performed a systematic search for studies reporting characteristics and outcome of patients with and without significant MR and/or adjusted mortality associated with MR post-TAVI. We conducted a meta-analysis of quantitative data. Results: Seventeen studies with 20,717 patients compared outcomes and group characteristics. Twenty-one studies with 32,257 patients reported adjusted odds of mortality associated with MR. Patients with MR were older, had a higher Society of Thoracic Surgeons score, lower left ventricular ejection fraction, a higher incidence of prior myocardial infarction, atrial fibrillation, and a trend towards higher NYHA class III/IV, but had similar mean gradient, gender, and chronic kidney disease. The MR patients had a higher unadjusted short-term (RR =
\end{abstract}

$1.46,95 \% \mathrm{Cl} 1.30-1.65)$ and long-term mortality $(\mathrm{RR}=1.40$, $95 \% \mathrm{Cl} 1.18-1.65)$. However, 16 of 21 studies with 27,777 patients found no association between MR and mortality after adjusting for baseline variables. In greater than half of the patients $(0.56,95 \% \mathrm{Cl} 0.45-0.66) \mathrm{MR}$ improved by at least one grade following TAVI. Conclusion: The patients with MR undergoing TAVI have a higher burden of risk factors which can independently impact mortality. There is a lack of robust evidence supporting an increased mortality in MR patients, after adjusting for other compounding variables. MR tends to improve in the majority of patients post-TAVI.

(c) 2020 S. Karger AG, Basel

\section{Background}

Aortic stenosis (AS) and mitral regurgitation (MR) are two common valve disorders that frequently coexist. Calcification of the mitral annulus, leaflets, and subvalvular apparatus, along with leaflet tethering secondary to pressure overload and concomitant coronary artery disease are factors implicated in the pathogenesis of MR in patients with severe AS [1]. Current guidelines recommend mitral valve repair or replacement in patients with severe

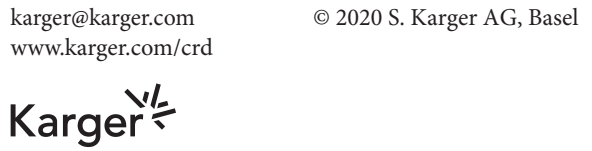

Ankur Sethi

125 Paterson Street, 6th floor

New Brunswick, NJ 08901 (USA)

drankursethi@gmail.com 
primary MR undergoing cardiac surgery for another indication [2]. The management of moderate MR in patients undergoing surgical aortic valve replacement (SAVR), and its mortality benefit, remains controversial $[3,4]$. The advent of transcatheter aortic valve implantation (TAVI) has resulted in a paradigm shift in the management of severe AS. Patients with significant MR were largely excluded from pivotal clinical trials. However, with the wider use of TAVI, concerns have been raised regarding the management of concomitant MR, affecting up to $20 \%$ of patients with severe AS [1].

Studies in patients with severe AS and significant MR, undergoing TAVI, have yielded mixed results [5-8]. Previous meta-analyses revealed higher short- and longterm mortality in patients with significant $\operatorname{MR}[9,10]$. Considering the observational nature of the studies, we postulated that patients with significant MR may have differences in baseline characteristics as compared to a control group devoid of significant MR, which would impact mortality. Therefore, the aim of our study is to compare the baseline characteristics of patients with and without significant MR and evaluate its impact on mortality in patients with severe AS undergoing TAVI.

\section{Methods}

A systematic search of the electronic databases PubMed and EMBASE was conducted for studies in patients with severe AS and MR undergoing TAVI reporting clinical outcomes. We used the following keywords "TAVR mitral regurgitation," "TAVI mitral regurgitation," "mitral regurgitation transcatheter aortic valve." We did not enforce any language restriction. We performed the last search on August 31, 2018. In addition, we searched the relevant websites acc.org and pcronline.com for the abstracts presented at major national meetings in the last 10 years. The bibliography of relevant review articles and prior meta-analysis were manually searched for any additional studies.

We included the studies which reported the outcomes of patients with and without MR before TAVI for AS and described the baseline characteristics of the two groups. In the event that a study did not describe group characteristics, it was included only if the mortality risk (odds or hazard ratio) associated with significant MR was adjusted for baseline variables using a multivariable regression. We excluded studies without any description of the characteristics or adjusted outcomes.

Two investigators independently searched the electronic databases for the relevant studies. We retrieved the full manuscripts of relevant studies for a detailed independent review. The data on study population, sites/registry, time period, definition of significant MR, duration of follow-up, post-procedure change in MR and timing of evaluation, group characteristics, short- and long-term mortality and adjusted mortality were extracted from the studies meeting the inclusion criteria. We selected those reports with a description of group characteristics, larger sample size, and longer follow-up, from the multiple reports extracted from the site or registry. A third author resolved any inconsistencies in the preliminary dataset. In the event of missing data, we contacted the corresponding author. This meta-analysis was conducted in accordance with the MOOSE (Meta-analysis of Observational Studies in Epidemiology) group recommendations [11].

\section{Statistical Analysis}

We extracted categorical and continuous characteristics to perform a quantitative meta-analysis of between-group differences in patients with and without significant MR using the mean difference and risk ratio (RR), respectively. Furthermore; a meta-analysis of unadjusted short- (30 days) and long-term mortality was performed as a function of the degree of significant MR. A subgroup analysis to assess the impact of significant MR on both the short- and long-term mortality was performed. A meta-analysis of adjusted hazard/odds ratio was planned, but not performed because of unreported effect size and confidence intervals in several studies with nonsignificant results. Because of the observational nature of the included studies and significant heterogeneity, we decided a priori to conduct a random effect meta-analysis, although heterogeneity among the studies for each outcome was assessed using the $\mathrm{I}^{2}$ statistic. The RR was used as summary statistic, and a $p$ value $<0.05$ was considered significant. Publication bias was assessed using the funnel plot and Egger method. All statistical analysis was conducted using "meta" package in the R statistical software (version 3.3.1) [12]. The quality of included studies was assessed using the Newcastle-Ottawa scale for non-randomized cohort studies [13].

\section{Results}

Seventeen studies with 20,717 patients compared the characteristics and outcomes of the groups with and without significant MR. Twenty-one studies reported the effect of significant MR on mortality adjusted for baseline variables. Figure 1 shows the flowchart depicting the search process. The characteristics of the included studies are shown in Table 1. Studies used grades as recommended by the American Society of Echocardiography/European Association of Echocardiography $[14,15]$ to define significant MR. Four studies used MR $\geq$ grade 3 (moderate to severe), one study used grade 4 (severe), and the rest used $\geq$ grade 2 (moderate) to define significant MR. From this point onwards in the study, we refer to patients with significant MR as MR patients and those without significant MR as patients no MR. The proportion of patients with pre-TAVI MR varied from 8 to $53 \%$ in all eligible studies, with follow-up ranging from 8 months to 5 years.

Patients with MR were: older (MR 82.4 years [81.483.3], without MR 81.3 years [80.7-81.93]); had higher Society of Thoracic Surgeons (STS) scores (MR [8.8, 95\% CI 7.9-9.8], without MR [7.6, 95\% CI 6.91-8.46]); de- 
Fig. 1. Studies selection process.

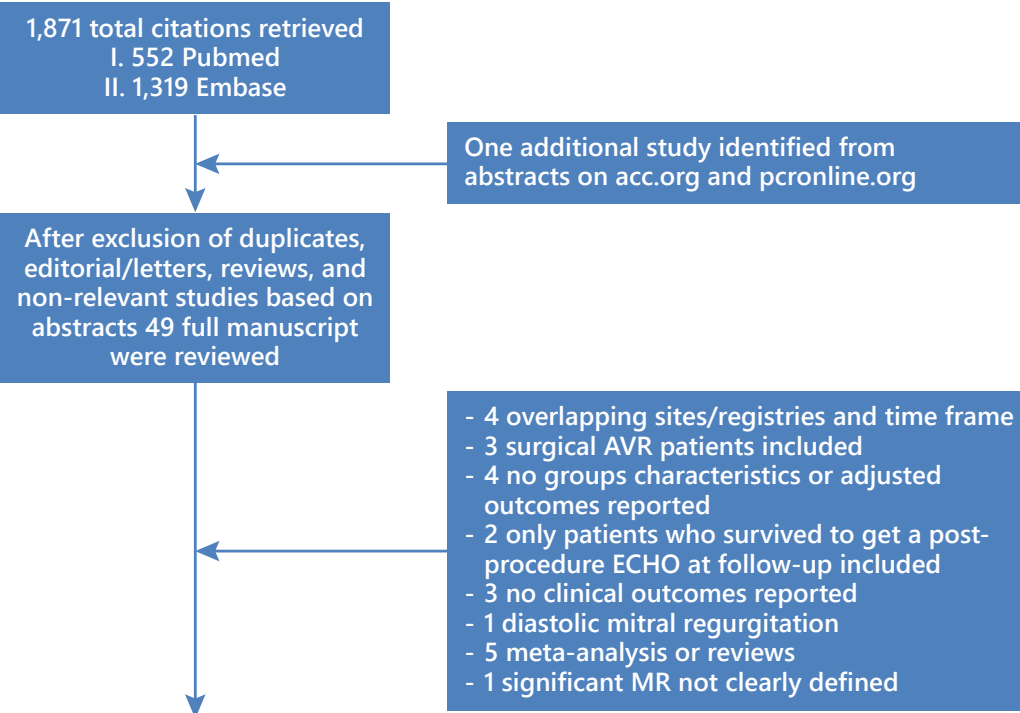

\section{6 studies included in}

the final analysis

Fig. 2. Forest plots showing difference between patients with and without significant MR (a), continuous variables, and categorical variables (b). AFIB, atrial fibrillation; CKD, chronic kidney disease; LVEF, left ventricle ejection fraction; MI, myocardial infarction; STS, Society of Thoracic Surgeons.

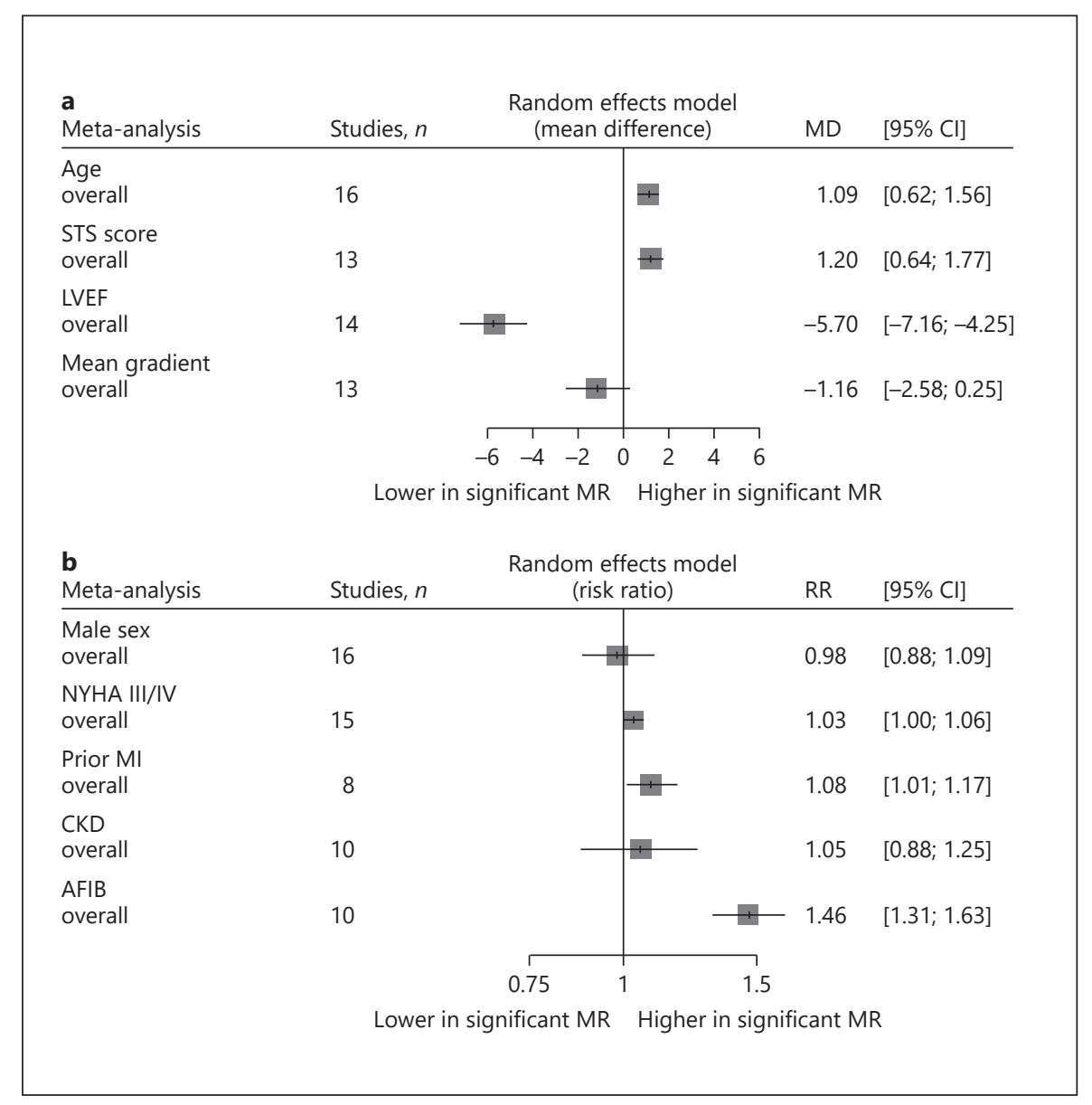




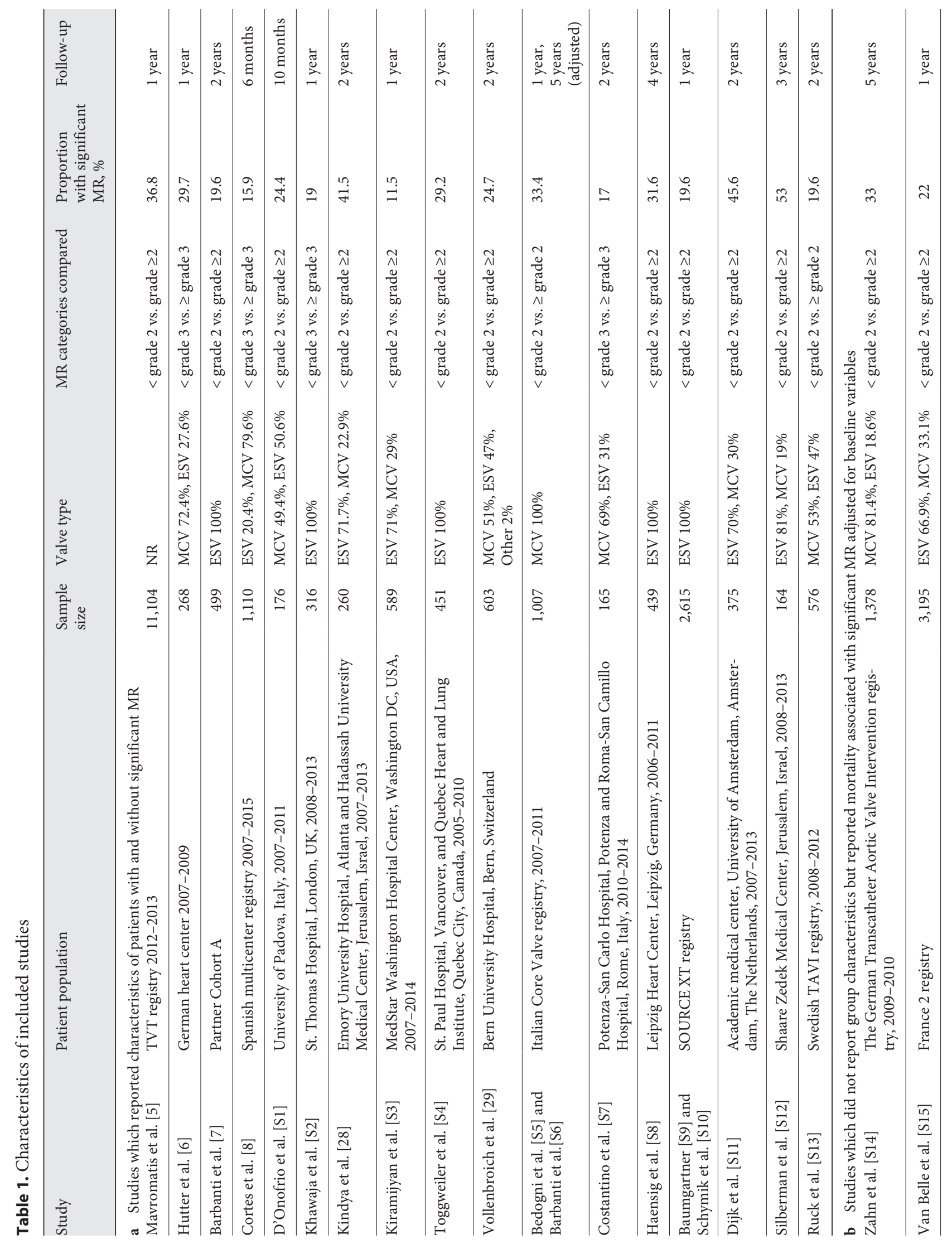




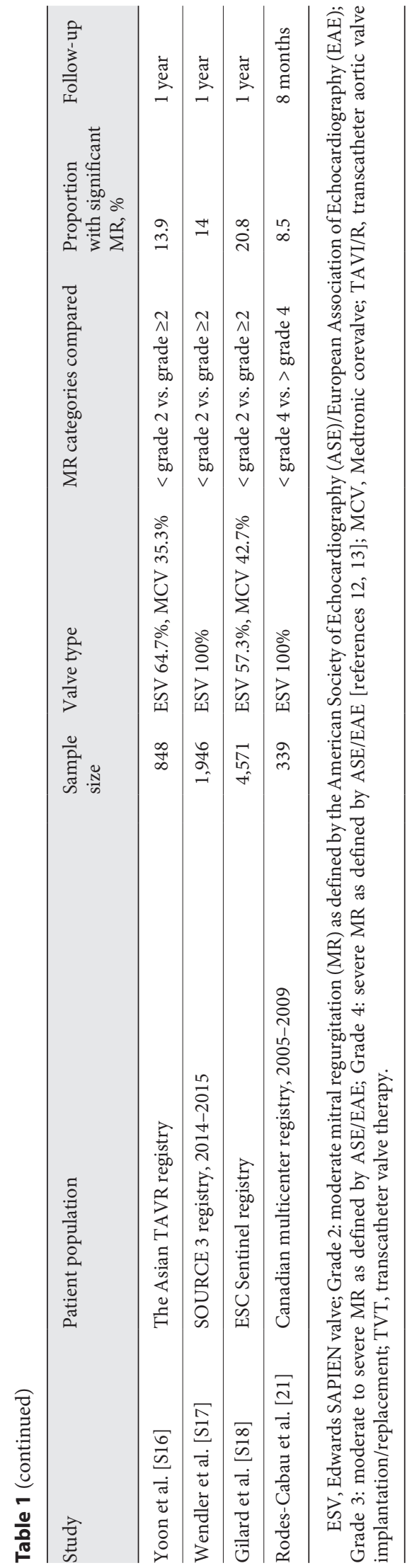

pressed left ventricular ejection fraction (MR [48.8\%, 95\% CI 46.8-50.8], no MR [54.7\%, 95\% CI 53.4-56]); more likely to have had a myocardial infarction MR (27\%, 95\% CI 21-33), no MR (23\%, 95\% CI 19-28)]; and, a higher incidence of atrial fibrillation (MR [37\%, 95\% CI 30-44], no MR [24\%, 95\% CI 19-30]). The mean gradient across the valve was similar (MR $44.7 \mathrm{~mm} \mathrm{Hg}$ [95\% CI 43.2-46.1] vs. no MR $45.9 \mathrm{~mm} \mathrm{Hg}$ [95\% CI 45.1-46.8]). There were $43.9 \%$ (95\% CI 40.7-44.6) and 45.6\% (95\% CI 42.5-48.7) males in the MR and no MR groups, respectively. In the MR and no MR groups, 78.9\% (95\% CI 7085.4) and 76.6\% (95\% CI 68.5-83.2) patients, respectively, had NYHA class III or IV symptoms. There was no difference in prevalence of chronic kidney disease (38\%, $95 \%$ CI $15-69$ vs. $34 \%$, 95\% CI 17-56). The forest plot of differences in group characteristics is shown in Figure 2.

\section{MR and Mortality}

The unadjusted short-term ( $\leq 30$ days $)(R R=1.46$, 95\% CI 1.30-1.65) and long-term $(\mathrm{RR}=1.40,95 \% \mathrm{CI}$ 1.18-1.65) mortality was higher in patients with $\mathrm{MR}$ compared to no MR as shown in Figures 3 and 4, respectively. There was no evidence interaction between the definition of significant MR, i.e., grade 2 versus grade 3, and unadjusted risk of mortality based on testing for subgroup difference as shown in Figures 3 and 4.

Twenty-one studies with 32,257 patients investigated an independent association between MR and mortality adjusted for other baseline variables. Twelve of the 21 studies reported effect size with a confidence interval, whereas the other 9 only commented on statistical insignificance. Sixteen of 21 studies with 27,777 patients found no association between MR and mortality, after adjusting for baseline variables (Fig. 5).

\section{Improvement in MR Post-TAVI}

Fourteen studies assessed 6,139 MR patients at followup with echocardiogram. The follow-up ranged from 1 month to 1 year. The improvement in MR by at least one grade was reported in 0.56 (95\% CI 0.45-0.66) proportion of patients (Fig. 6). Predictors of persistent MR post-TAVI are also summarized in Figure 6.

\section{Publication Bias and Quality Assessment}

We found no evidence of publication bias on visual inspection of the funnel plot or by the Egger method (short-term mortality $p=0.42$, long-term mortality $p=$ $0.67)$ as shown in online supplementary Figure S1 and Figure S2 (for all online suppl. material, see www. karger.com/doi/10.1159/000506624). We did not find 


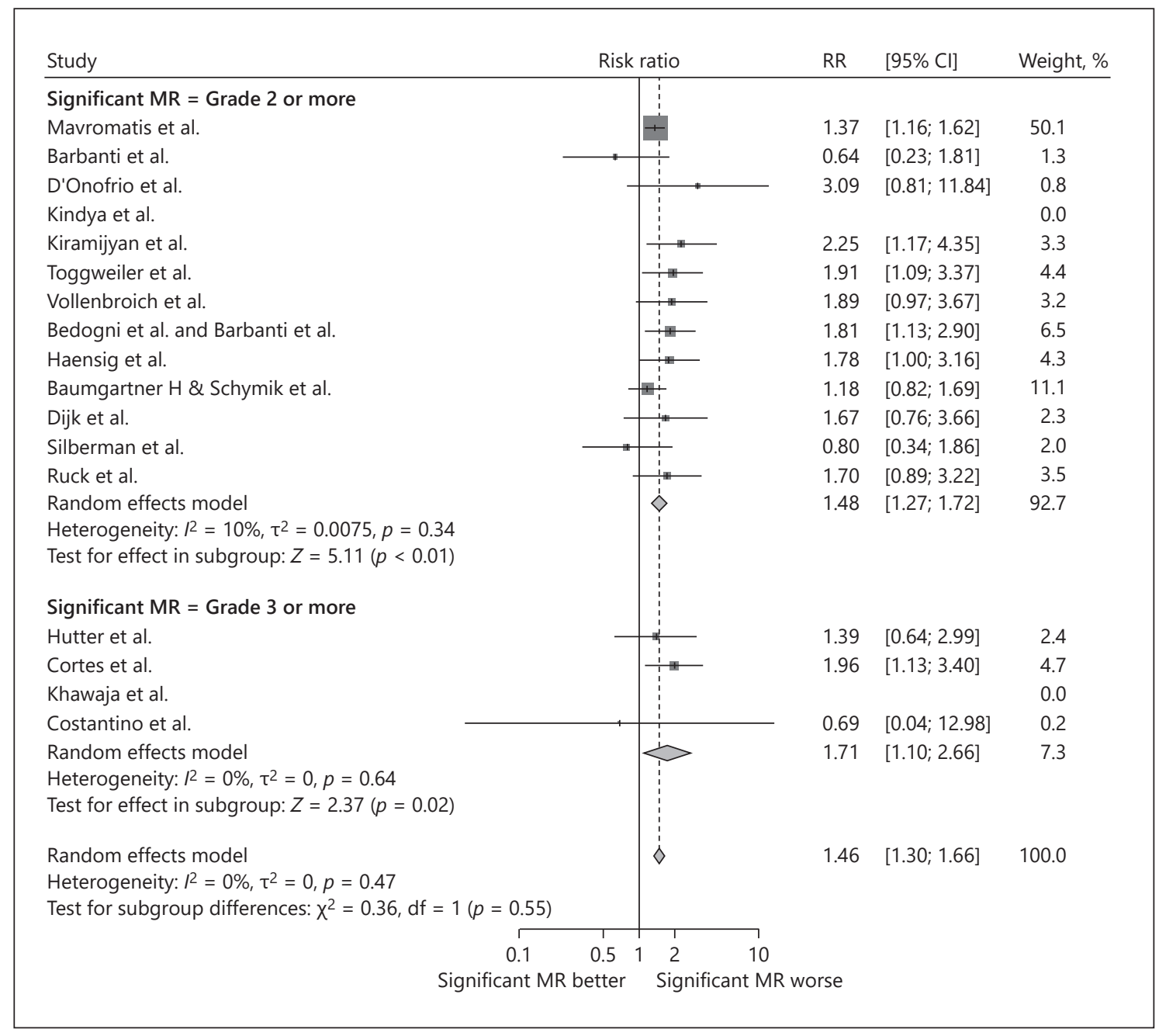

Fig. 3. Forest plot showing unadjusted RR of short-term mortality ( $\leq 30$ days).

bias in selection of subjects based on four subcategories of the Newcastle-Ottawa scale. The groups were different in two or more tested characteristics in all included studies as shown in online supplementary Table S1. We considered studies with $\geq 1$ year follow-up to have been of sufficient follow-up duration, with $>90 \%$ of studies meeting these criteria. The assessment of study quality using this scale is shown in online supplementary Table S1.

\section{Discussion}

We conducted a quantitative meta-analysis and systematic review of all available evidence to evaluate the association between significant preprocedural MR and the risk of mortality after TAVI, for severe AS. In addition, we studied the baseline characteristic differences between patients with and without MR which may affect clinical outcomes. In an unadjusted analysis, MR was associated with a higher short- and long-term mortality; however, significant differences in baseline characteristics between the two groups were noted. A systematic review of prior studies assessing the presence of significant MR on mortality, adjusted for baseline characteristics, showed no association. This is in contradistinction to three prior metaanalyses, of 8-13 studies including $\leq 9,000$ patients, which found a higher short- and long-term mortality in patients with MR $[9,10,16]$.

Nombela-Franco et al. [9] performed a meta-analysis ( $n=8,015$ patients) and reported a higher adjusted mortality in those patients with MR. There were several differences compared to our analysis including a smaller 


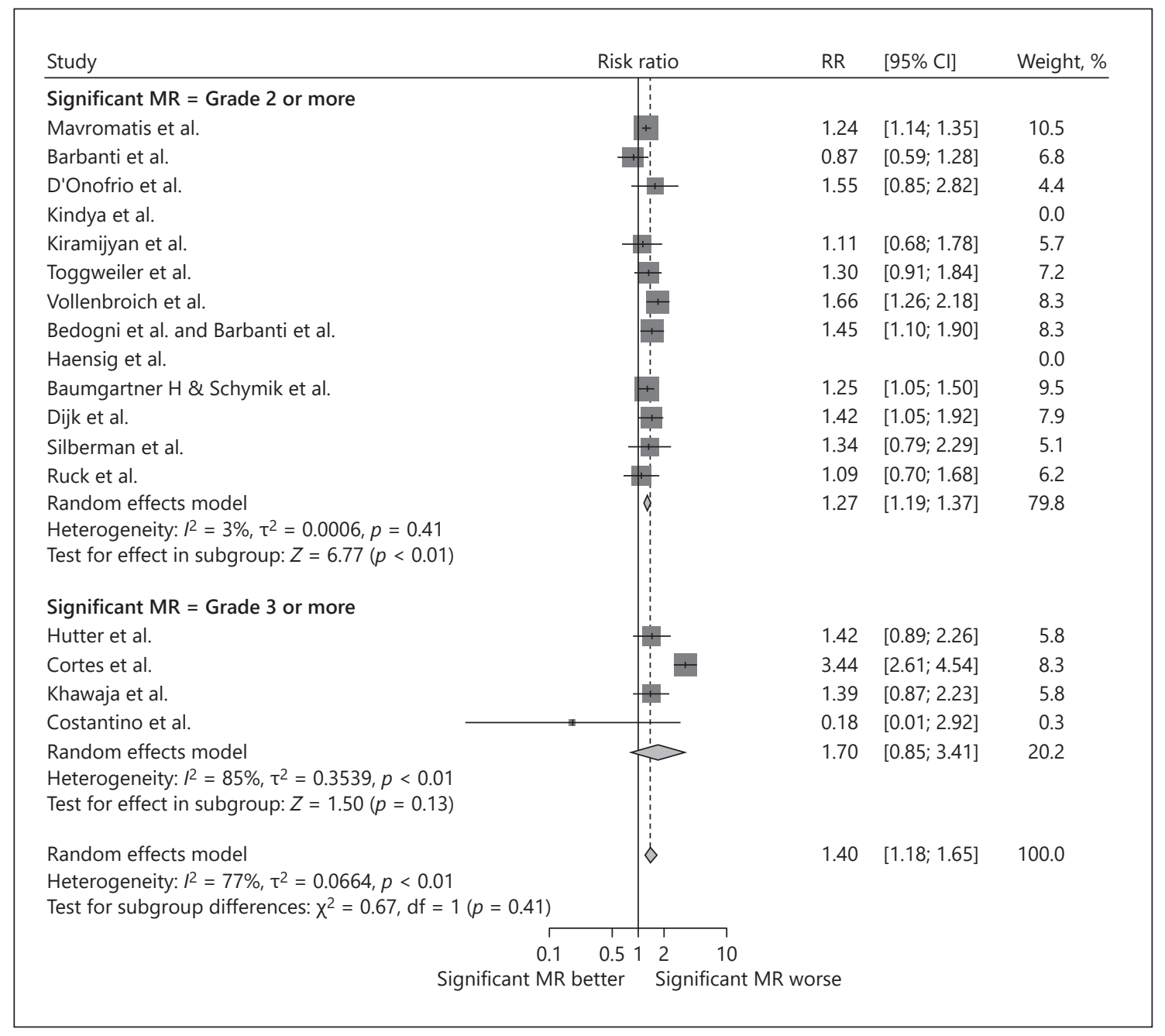

Fig. 4. Forest plot showing unadjusted RR of long-term mortality.

number of patients per study while restricting inclusion to national registries and randomized clinical trials. Other investigators reported significant MR in only $63 \%$ of patients, did not include this variable as a predictor of mortality in their original manuscript [17], but, included an adjusted odds ratio in their meta-analysis [9]. We paid special attention to the site and time frame of patient recruitment to prevent data duplication. More importantly, we found that many studies with no significant relationship between MR and adjusted mortality failed to report an effect size and confidence interval (Fig. 5), introducing substantial bias. Therefore, we decided not to perform a quantitative synthesis for adjusted odds ratio and opted to report qualitative outcomes.

In contrast to prior meta-analyses, we concluded that patients with MR exhibit significant baseline differences compared to controls, which may independently impact mortality. In a meta-analysis of 11 studies $(n>11,000$ patients), the presence of atrial fibrillation was associated with a higher short- and long-term mortality in patients undergoing TAVI [18]. Similarly, a lower LVEF and a higher STS score were associated with a higher mortality following TAVI $[19,20]$. In this analysis, patients with concomitant MR were older, had a higher STS score, lower LVEF, had higher incidence of prior MI, and atrial fibrillation. Although not reaching statistical significance, patients with MR had a higher NYHA heart failure class. There were no differences in gender, incidence of chronic kidney disease, and the mean gradient compared to the no MR group.

These variables were selected based on their uniform availability from the extracted studies. Several other 


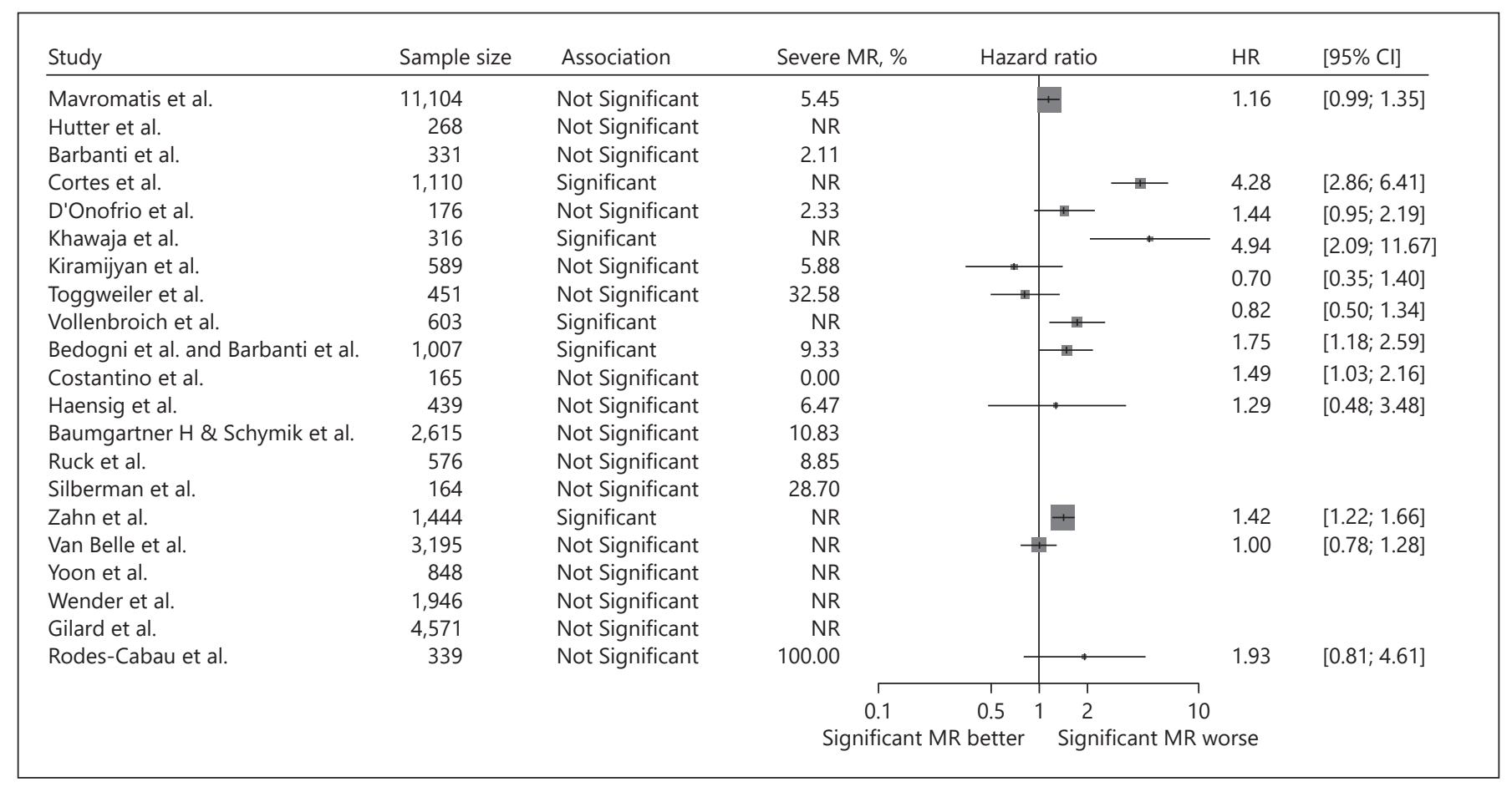

Fig. 5. Forest plot showing results adjusted odds/hazards of mortality associated with significant MR including studies which did not report effect size.

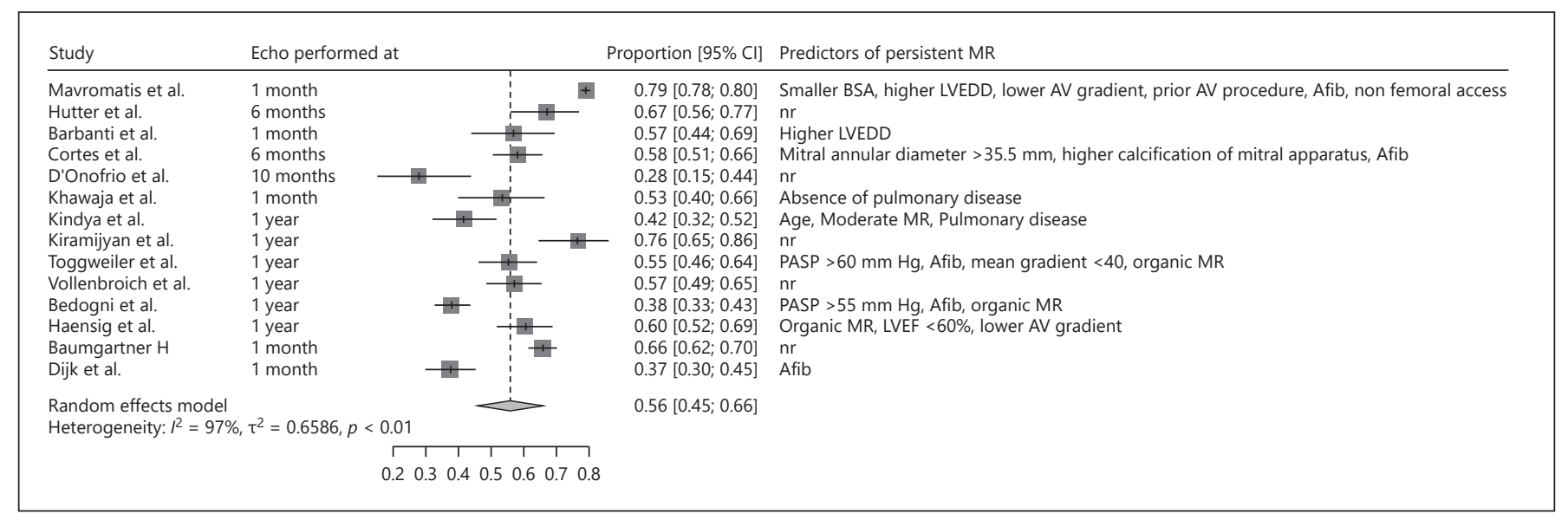

Fig. 6. Forest plot showing improvement in MR by at least 1 grade among the included studies.

variables which may impact mortality were unbalanced and not incorporated in the analysis (see online suppl. Table S1). Considering the observational nature of all available evidence, we focused on studies which adjusted for baseline variables, when assessing the association between MR and mortality. As shown in Figure 5, most studies did not show an association between MR and mortality when adjusted for other variables. There are several possible explanations for this observation: with the exception of the study conducted by Rodés-Cabau et al. [21], which only included patients with severe MR, only 1 of 3 patients enrolled had severe MR; only four studies had a follow-up period $>2$ years, with only an 8-month follow-up in the Rodés-Cabau study [21]. The mortality in the TAVR groups at 2 years in Partner $1 \mathrm{~A}$ and $1 \mathrm{~B}$ were 33.9 and $43.3 \%$, respectively, with a signifi- 
cant proportion of death attributed to noncardiac causes $[22,23]$. Therefore, it was not surprising that MR had no impact on mortality in patients with moderate MR, during short-term follow-up, and with other high-risk cardiovascular as well as noncardiovascular comorbidities. Secondly, in the majority of patients, the MR improved by at least a grade on the follow-up, supporting the hypothesis of significant MR being a risk marker, as unadjusted mortality was higher in both short and long term. Finally, it is interesting to note that MR is not an independent predictor of mortality in commonly used risk scores, which adjust for multiple variables, like STS score and EuroScore II after SAVR [24, 25] or TAVR specific risk scores $[26,27]$.

We found that MR improved post-TAVI at follow-up in $56 \%$ of patients (Fig. 6), consistent with previous studies $[9,10]$. The reduction in gradient across the mitral valve due to the instantaneous decrease in left ventricle pressure after resolution of AS post-TAVI and continuous reverse remodeling of the left ventricle has been postulated to promote MR improvement acutely and upon follow-up [1]. Interestingly, measures of left ventricle size, severe pulmonary hypertension, presence of organic $\mathrm{MR}$, atrial fibrillation, and lower aortic valve gradient were associated with persistence of MR in several studies. This may be a consequence of a severely dilated left ventricle and lower aortic valve gradient representing primary myocardial disease; thus, the afterload reduction post-TAVI may not significantly improve MR, similar to patients with underlying organic mitral valve pathology like prolapse or a flailed leaflet. Likewise, the presence of atrial fibrillation and severe pulmonary hypertension may represent a more advanced stage of mitral valve dysfunction, as well as, persistent pulmonary vascular remodeling. Further characterization of predictors of persistence of MR post-TAVI in future studies may help with decision-making and counselling of patients with MR and severe AS referred for TAVI.

There are several unresolved issues. The impact of functional versus organic MR on outcomes is not well understood. Two studies in the analysis compared outcomes of patients with these two entities [28, 29]. Kindya et al. [28] reported that patients with moderate to severe organic MR were less likely to have a survival free of death or hospitalization on multivariable analysis, whereas functional MR was not an independent predictor. Vollenbroich et al. [29] found organic MR to be independently associated with cardiovascular mortality at 2 years and not the functional MR. On the other hand, Kiramijyan et al. [30] found no difference in mortality between organic and functional MR at 30 days and 1 year; although there was a larger improvement in the severity of $M R$, LVEF, and symptoms in patients with functional MR. Previous meta-analyses did not find any difference on the impact of MR and mortality as a function of the type of TAVI valve used (e.g., balloon expandable versus self-expanding) $[9,10]$. These studies were not randomized; therefore, the role of prosthesis selection and patient specific variables could not be analyzed.

\section{Limitations}

This was an analysis based on observational studies, a prospective study analyzing prospectively gathered data would have allowed for adjustment of all confounders. There was significant heterogeneity across the studies, as shown by $I^{2}$ statistic; therefore, random effect analysis was performed. As noted above, only a limited number of patients had severe MR, consequently, more information on this group of patients is needed. In addition, the impact of the etiology of MR, functional, organic or mixed, on outcomes remains unclear. In only three studies, MR was evaluated by an independent core laboratory or echocardiographer, and only four studies have outcomes adjudicated by an independent committee. The impact of blind adjudication committee on mortality, an unambiguous outcome, is probably minimal. On the other hand, echocardiographic assessment of MR severity is challenging not only due to calcification, but also large color Doppler jet secondary to high trans-mitral driving velocity frequently seen in severe AS [1,31]. Therefore, a blinded core lab assessment of MR severity both preprocedure and on follow-up may significantly affect the results.

\section{Conclusion}

In a comprehensive meta-analysis of observational studies, we found that patients with greater than moderate MR in severity exhibited a higher mortality post-TAVI. However, we found significant differences in the baseline characteristics of the two groups which may independently contribute to this difference in mortality. Current evidence does not support moderate MR as an independent mortality risk factor post-TAVI. We conclude from this analysis that, at the present time, moderate to severe MR may not be a contraindication for TAVI in an otherwise appropriate patient with severe AS, since the majority of patients exhibit improvement in MR at follow-up. 


\section{Disclosure Statement}

We do not have any conflicts of interest related to this study.

\section{Funding Sources}

No funding was received.

\section{References}

1 Sannino A, Grayburn PA. Mitral regurgitation in patients with severe aortic stenosis: diagnosis and management. Heart. 2018 Jan; 104(1):16-22.

2 Nishimura RA, Otto CM, Bonow RO, Carabello BA, Erwin JP 3rd, Guyton RA, et al.; ACC/AHA Task Force Members. 2014 AHA/ ACC Guideline for the Management of Patients With Valvular Heart Disease: a report of the American College of Cardiology/ American Heart Association Task Force on Practice Guidelines. Circulation. 2014 Jun; 129(23):e521-643.

3 Alghamdi AA, Elmistekawy EM, Singh SK, Latter DA. Is concomitant surgery for moderate functional mitral regurgitation indicated during aortic valve replacement for aortic stenosis? A systematic review and evidencebased recommendations. J Card Surg. 2010 Mar;25(2):182-7.

4 Harling L, Saso S, Jarral OA, Kourliouros A, Kidher E, Athanasiou T. Aortic valve replacement for aortic stenosis in patients with concomitant mitral regurgitation: should the mitral valve be dealt with? Eur J Cardiothorac Surg. 2011 Nov; 40(5):1087-96.

5 Mavromatis K, Thourani VH, Stebbins A, Vemulapalli S, Devireddy C, Guyton RA, et al. Transcatheter Aortic Valve Replacement in Patients With Aortic Stenosis and Mitral Regurgitation. Ann Thorac Surg. 2017 Dec; 104(6):1977-85.

6 Hutter A, Bleiziffer S, Richter V, Opitz A, Hettich I, Mazzitelli D, et al. Transcatheter aortic valve implantation in patients with concomitant mitral and tricuspid regurgitation. Ann Thorac Surg. 2013 Jan;95(1):77-84.

7 Barbanti M, Webb JG, Hahn RT, Feldman T, Boone RH, Smith CR, et al.; Placement of Aortic Transcatheter Valve Trial Investigators. Impact of preoperative moderate/severe mitral regurgitation on 2-year outcome after transcatheter and surgical aortic valve replacement: insight from the Placement of Aortic Transcatheter Valve (PARTNER) Trial Cohort A. Circulation. 2013 Dec;128(25): 2776-84.

8 Cortés C, Amat-Santos IJ, Nombela-Franco L, Muñoz-Garcia AJ, Gutiérrez-Ibanes E, De La Torre Hernandez JM, et al. Mitral Regurgitation After Transcatheter Aortic Valve Replacement: Prognosis, Imaging Predictors, and Potential Management. JACC Cardiovasc Interv. 2016 Aug;9(15): 1603-14.

9 Nombela-Franco L, Eltchaninoff H, Zahn R, Testa L, Leon MB, Trillo-Nouche R, et al. Clinical impact and evolution of mitral regur- gitation following transcatheter aortic valve replacement: a meta-analysis. Heart. 2015 Sep;101(17):1395-405.

10 Chakravarty T, Van Belle E, Jilaihawi H, Noheria A, Testa L, Bedogni F, et al. Meta-analysis of the impact of mitral regurgitation on outcomes after transcatheter aortic valve implantation. Am J Cardiol. 2015 Apr;115(7): 942-9.

11 Stroup DF, Berlin JA, Morton SC, Olkin I, Williamson GD, Rennie D, et al. Meta-analysis of observational studies in epidemiology: a proposal for reporting. Meta-analysis Of Observational Studies in Epidemiology (MOOSE) group. JAMA. 2000 Apr;283(15): 2008-12.

12 Schwarzer G. meta: an R package for metaanalysis. R News. 2007;7(3):40-5.

13 Wells GA, Shea B, O'connell D, Peterson J, Welch V. The Newcastle-Ottawa Scale (NOS) for assessing the quality of nonrandomised studies in meta-analyses. 2000. Available from: www.ohri.ca/programs/clinical_epidemiology/oxford.asp. (Accessed: 20 September, 2018).

14 Lancellotti P, Moura L, Pierard LA, Agricola E, Popescu BA, Tribouilloy C, et al.; European Association of Echocardiography. European Association of Echocardiography recommendations for the assessment of valvular regurgitation. Part 2: mitral and tricuspid regurgitation (native valve disease). Eur J Echocardiogr. 2010 May;11(4):307-32.

15 Zoghbi WA, Enriquez-Sarano M, Foster E, Grayburn PA, Kraft CD, Levine RA, et al.; American Society of Echocardiography. Recommendations for evaluation of the severity of native valvular regurgitation with two-dimensional and Doppler echocardiography. J Am Soc Echocardiogr. 2003 Jul;16(7):777802.

16 Sannino A, Losi MA, Schiattarella GG, Gargiulo G, Perrino C, Stabile E, et al. Meta-analysis of mortality outcomes and mitral regurgitation evolution in 4,839 patients having transcatheter aortic valve implantation for severe aortic stenosis. Am J Cardiol. 2014 Sep; 114(6):875-82.

17 Sabaté M, Cánovas S, García E, Hernández Antolín R, Maroto L, Hernández JM, et al.; collaborators of the TAVI National Group. In-hospital and mid-term predictors of mortality after transcatheter aortic valve implantation: data from the TAVI National Registry 2010-2011. Rev Esp Cardiol (Engl Ed). 2013 Dec;66(12):949-58.

18 Mojoli M, Gersh BJ, Barioli A, Masiero G, Tellaroli P, D'Amico G, et al. Impact of atrial fi- brillation on outcomes of patients treated by transcatheter aortic valve implantation: A systematic review and meta-analysis. Am Heart J. 2017 Oct;192:64-75.

19 Sannino A, Gargiulo G, Schiattarella GG, Brevetti $\mathrm{L}$, Perrino $\mathrm{C}$, Stabile E, et al. Increased mortality after transcatheter aortic valve implantation (TAVI) in patients with severe aortic stenosis and low ejection fraction: a metaanalysis of 6898 patients. Int J Cardiol. 2014 Sep;176(1):32-9.

20 Hemmann K, Sirotina M, De Rosa S, Ehrlich JR, Fox H, Weber J, et al. The STS score is the strongest predictor of long-term survival following transcatheter aortic valve implantation, whereas access route (transapical versus transfemoral) has no predictive value beyond the periprocedural phase. Interact Cardiovasc Thorac Surg. 2013 Aug; 17(2):359-64.

21 Rodés-Cabau J, Webb JG, Cheung A, Ye J, Dumont E, Feindel CM, et al. Transcatheter aortic valve implantation for the treatment of severe symptomatic aortic stenosis in patients at very high or prohibitive surgical risk: acute and late outcomes of the multicenter Canadian experience. J Am Coll Cardiol. 2010 Mar; 55(11):1080-90.

22 Kodali SK, Williams MR, Smith CR, Svensson LG, Webb JG, Makkar RR, et al.; PARTNER Trial Investigators. Two-year outcomes after transcatheter or surgical aortic-valve replacement. N Engl J Med. 2012 May;366(18):168695.

23 Makkar RR, Fontana GP, Jilaihawi H, Kapadia S, Pichard AD, Douglas PS, et al.; PARTNER Trial Investigators. Transcatheter aortic-valve replacement for inoperable severe aortic stenosis. N Engl J Med. 2012 May; 366(18):1696-704.

24 O'Brien SM, Shahian DM, Filardo G, Ferraris VA, Haan CK, Rich JB, et al.; Society of Thoracic Surgeons Quality Measurement Task Force. The Society of Thoracic Surgeons 2008 cardiac surgery risk models: part 2-isolated valve surgery. Ann Thorac Surg. 2009 Jul;88(1 Suppl):S23-42.

25 Nashef SA, Roques F, Sharples LD, Nilsson J, Smith C, Goldstone AR, et al. EuroSCORE II. Eur J Cardiothorac Surg. 2012 Apr;41(4): 734-44.

26 Hermiller JB Jr, Yakubov SJ, Reardon MJ, Deeb GM, Adams DH, Afilalo J, et al.; CoreValve United States Clinical Investigators. Predicting Early and Late Mortality After Transcatheter Aortic Valve Replacement. J Am Coll Cardiol. 2016 Jul;68(4): 343-52. 
27 Edwards FH, Cohen DJ, O’Brien SM, Peterson ED, Mack MJ, Shahian DM, et al.; Steering Committee of the Society of Thoracic Surgeons/American College of Cardiology Transcatheter Valve Therapy Registry. Development and Validation of a Risk Prediction Model for In-Hospital Mortality After Transcatheter Aortic Valve Replacement. JAMA Cardiol. 2016 Apr;1(1):46-52.
28 Kindya B, Ouzan E, Lerakis S, Gonen E, Babaliaros V, Karayel E, et al. Degenerative mitral regurgitation predicts worse outcomes in patients undergoing transcatheter aortic valve replacement. Catheter Cardiovasc Interv. 2018 Sep;92(3):574-82.

29 Vollenbroich R, Stortecky S, Praz F, Lanz J, Franzone A, Zuk K, et al. The impact of functional vs degenerative mitral regurgitation on clinical outcomes among patients undergoing transcatheter aortic valve implantation. Am Heart J. 2017 Feb;184:71-80.
30 Kiramijyan S, Koifman E, Asch FM, Magalhaes MA, Didier R, Escarcega RO, et al. Impact of Functional Versus Organic Baseline Mitral Regurgitation on Short- and LongTerm Outcomes After Transcatheter Aortic Valve Replacement. Am J Cardiol. 2016 Mar; 117(5):839-46.

31 Grayburn PA, Weissman NJ, Zamorano JL. Quantitation of mitral regurgitation. Circulation. 2012 Oct;126(16):2005-17. 